\title{
Using sentinels to detect intersections of convex and nonconvex polygons
}

\author{
W.F. MASCARENHAS and E.G. BIRGIN \\ Computer Science Department, Institute of Mathematics and Statistics \\ University of São Paulo, Rua do Matão, 1010, Cidade Universitária \\ 05508-090 São Paulo, SP, Brazil \\ E-mails: walterfm@ime.usp.br/ egbirgin@ime.usp.br
}

\begin{abstract}
We describe finite sets of points, called sentinels, which allow us to decide if isometric copies of polygons, convex or not, intersect. As an example of the applicability of the concept of sentinel, we explain how they can be used to formulate an algorithm based on the optimization of differentiable models to pack polygons in convex sets.
\end{abstract}

Mathematical subject classification: $90 \mathrm{C} 53,65 \mathrm{~K} 05$.

Key words: sentinels, polygons, intersection, packing, nonlinear programming.

\section{Introduction}

In [1] we propose a nonlinear programming approach to pack arbitrary polygons in convex sets (not necessarily polygons). This approach is based on the observation that if the interior of translated and rotated copies $P^{\prime}$ and $Q^{\prime}$ of the polygons $P$ and $Q$ in Figure 1 intersect then either the interior of $P^{\prime}$ contains one of the points $q_{i}^{\prime}$ or the interior of $Q^{\prime}$ contains some $p_{i}^{\prime}$. Motivated by this fact, we say that the $p_{i}$ and $q_{i}$ are sentinels for $\{P, Q\}$ with respect to translations and rotations.

The observation above leads to the following algorithm to pack translated and rotated copies of $P_{1}, P_{2}, \ldots, P_{N}$ on a convex set $C$ : 

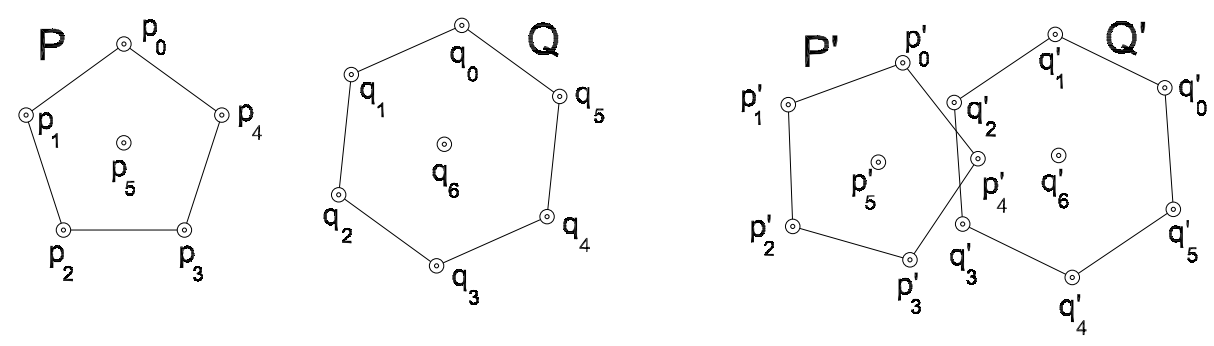

Figure 1 - The points $p_{i}$ and $q_{i}$ are sentinels: they detect if translated and rotated copies of the interior of $P$ and $Q$ intersect. In this example $p_{4}^{\prime}$ is in the interior of $Q^{\prime}$.

1. We parameterize $P_{i}$ by the coordinates $\left(x_{i}, y_{i}\right)$ of its barycenter and its rotation angle $\theta_{i}$.

2. We define differentiable functions $\Psi_{i j}\left(x_{i}, y_{i}, \theta_{i}, x_{j}, y_{j}, \theta_{j}\right)$ based on the distance of the sentinels of $P_{i}$ to $P_{j}$ so that $\Psi_{i j}\left(x_{i}, y_{i}, \theta_{i}, x_{j}, y_{j}, \theta_{j}\right)$ is zero if all the sentinels of $P_{i}$ are outside the interior of $P_{j}$ and $\Psi_{i j}\left(x_{i}, y_{i}, \theta_{i}, x_{j}, y_{j}, \theta_{j}\right)$ is positive otherwise. (See [1] for an example of $\Psi_{i j}$-functions for identical rectangles.)

3. We find approximations to the solution of the feasibility problem

$$
\Psi_{i j}\left(x_{i}, y_{i}, \theta_{i}, x_{j}, y_{j}, \theta_{j}\right)=0 \quad \text { and } \quad \text { vertices of } P_{i} \subset C \text {. }
$$

For each solution of problem (1) we obtain a packing of $P_{1}, P_{2}, \ldots, P_{N}$ in $C$.

In [1] we describe the nonlinear programming aspects of the approach above in detail, from the theoretical and practical perspectives. In $[5,7,8]$, Stoyan's $\Phi$-functions are introduced. A $\Phi$-function for a pair of polygons is defined as a function whose value is positive if the polygons overlap and zero otherwise. Our functions $\Psi$ are analogous to Stoyan's $\Phi$-functions in the sense that they are $\Phi$-functions defined through the usage of sentinels. Sentinels can also be used to detect the intersection of rotated and translated copies of polygons [6]. Finally, the concept of sentinel leads to packings which are not necessarily lattice-like [3].

In the present work we focus on the geometric aspects of the novel concept of sentinel. We formalize this concept for arbitrary families of polygons and 
discuss their existence and complexity. Sentinel is a neat concept, but unfortunately some polygons require an infinite number of them. For instance, if instead of the polygons $P$ and $Q$ in Figure 1 we consider translated and rotated copies of a single triangle $T$ then no finite set of points $\left\{t_{1}, \ldots, t_{n}\right\}$, no matter how large, would be enough to detect all the intersections of $T$ and a translated copy $T^{\prime}$ of it: see Figure 2; we can always translate $T^{\prime}$ (maintaining the overlapping with $T$ ) in order to have $t_{c}^{\prime}$ outside $T$ and $t_{a}$ outside of $T^{\prime}$ no matter how near we define $t_{a}$ from $t_{b}$ and $t_{c}^{\prime}$ from $t_{d}^{\prime}$.

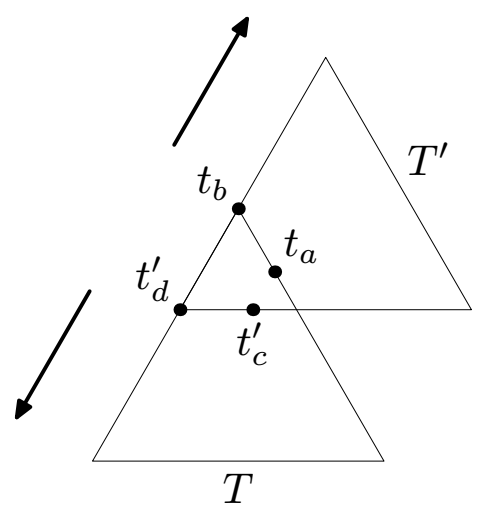

Figure 2 - There are no finite sets of sentinels for triangles. No matter how near we define $t_{a}$ and $t_{b}$ for $T$ and $t_{c}^{\prime}$ and $t_{d}^{\prime}$ for $T^{\prime}$, it will always be possible to overlap $T$ and $T^{\prime}$ having $t_{c}^{\prime} \notin T$ and $t_{a} \notin T^{\prime}$.

Small internal angles are the main reason why we may need an infinite number of sentinels. More precisely, in the next section we show that if $\mathcal{P}$ is a finite family of convex and non-convex polygons $P$ such that all the internal angles of $P$ are bigger than or equal to $\pi / 2$ then we can assign a finite set $S(P) \subset \mathbb{R}^{2}$ to each $P \in \mathcal{P}$ in such way that if $T, U: \mathbb{R}^{2} \rightarrow \mathbb{R}^{2}$ are isometries, $P, Q \in \mathcal{P}$ and the interior of $T(P)$ and $U(Q)$ intersect then either $T(S(P))$ intersects the interior of $U(Q)$ or $U(S(Q))$ intersects the interior of $T(P)$. In Section 2 we also define the terms we use throughout the paper and present basic results about the existence of sentinels. In Section 3 we discuss sentinel assignments for rectangles, which are the main motivation behind [1]. We present minimal sets of sentinels for the families of rectangles that motivated [1] and lead us to define the concept of sentinel. The last section contains concluding remarks. 


\section{Sentinels}

In this section we formalize the concept of sentinel and prove the existence of finite sets of sentinels for relevant families of polygons. We start by defining the basic terms we use:

Notation 1. If $u, v \in \mathbb{R}^{2}$ then we call the segment with extremes $u$ and $v$ by $u v$.

Convention 1. A polygon $P$ is defined in terms of an integer $n \geq 3$ and vertices $p_{i} \in \mathbb{R}^{2}$, for $i \in \mathbb{Z}$, such that

(a) $p_{i+n}=p_{i}$ for all $i$ and $p_{i+j} \neq p_{i}$ if $j$ is not a multiple of $n$.

(b) $p_{i} \notin p_{i-1} p_{i+1}$ for all $i$.

(c) If the segments $p_{i} p_{i+1}$ and $p_{j} p_{j+1}$ intersect then either

(i) $i \equiv j \bmod n$, or

(ii) $i \equiv j+1 \bmod n$ and $p_{i} p_{i+1} \cap p_{j} p_{j+1}=\left\{p_{i}\right\}$, or

(iii) $i+1 \equiv j \bmod n$ and $p_{i} p_{i+1} \cap p_{j} p_{j+1}=\left\{p_{j}\right\}$.

These conditions imply that $P$ is a Jordan curve. We denote the interior of this curve by $\operatorname{int}(P)$ and its border by border $(P)$. Moreover, we assume that the points $p_{i}$ are in counterclockwise order and use $n(P)$ to denote $n$.

Notation 2. $\operatorname{Sub}\left(\mathbb{R}^{2}\right)$ denotes the set whose elements are the subsets of $\mathbb{R}^{2}$.

Using this terminology we can formalize the concept of sentinel:

Definition 1. Let $\mathcal{P}$ be a family of polygons and let $\mathcal{T}$ be a family of transformations $T: \mathbb{R}^{2} \rightarrow \mathbb{R}^{2}$ such that $T(P)$ is a polygon for all $P \in \mathcal{P}$. We say that a function $S: \mathcal{P} \rightarrow \operatorname{Sub}\left(\mathbb{R}^{2}\right)$ is a sentinel assignment for $\mathcal{P}$ with respect to $\mathcal{T}$ if for all $T, U \in \mathcal{T}$ and $P, Q \in \mathcal{P}$ such that $\operatorname{int}(T(P)) \cap \operatorname{int}(U(Q)) \neq \emptyset$ we have that either $\operatorname{int}(T(P)) \cap U(S(Q)) \neq \emptyset$ or $\operatorname{int}(U(Q)) \cap T(S(P)) \neq \emptyset$. In this context, we say that the elements of $S(P)$ are the sentinels of $P$.

In this paper we care only about two families $\mathcal{T}$ of transformations: 
Notation 3. The isometries of $\mathbb{R}^{2}$ are transformations of the form $T(x)=$ $Q x+d$, where $Q$ is a $2 \times 2$ orthogonal matrix and $d \in \mathbb{R}^{2}$. We call the set of such isometries by $I_{2}$. We define $I_{2}^{+}$as the set of transformations $T$ above with $\operatorname{det} Q=1$.

In this section we provide sufficient conditions on the family $\mathcal{P}$ of polygons to guarantee the existence of a sentinel assignment $S$ for $\mathcal{P}$ with respect to $I_{2}$ such that $S(P)$ is finite for all $P \in \mathcal{P}$. In the next section we discuss the analogous question when $\mathcal{P}$ is a family of rectangles and the family of transformations is $I_{2}^{+}$. These conditions are expressed in terms of the parameter $\alpha(P)$ :

Notation 4. We call the smallest internal angle of the polygon $P$ by $\alpha(P)$.

The relevance of $\alpha(P)$ is illustrated in Figure 3. According to this figure, we can decide whether $b$ is inside, over or outside the circle with diameter $a c$ by looking at the angle $\alpha$. This figure is the motivation for the condition $\alpha(P) \geq \pi / 2$ used throughout this paper.
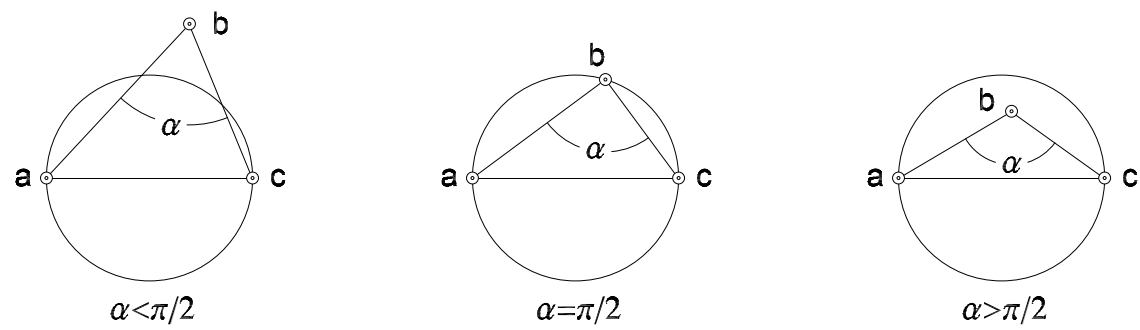

Figure 3 - The angle $\alpha$ and the position of $b$ with respect to the circle with diameter $a c$.

Besides $\alpha(P)$ our results are formulated using the sets $\Delta(P)$ :

Definition 2. Let $P$ be a polygon with $n \geq 4$ vertices (remember that we are dealing with convex and nonconvex polygons). We define $\Delta(P)$ as the set formed by the positive $\delta$ 's such that every segment uv connecting disjoint sides of $P$ has length bigger than $\delta$.

Unfortunately, $\Delta(P)$ is not as simple as $\alpha(P)$, starting with the fact that it is a set and not a number. Please, pay much attention to this fact, because it does not follow the computational geometry tradition of using numbers to characterize 
properties of polygons. For instance, the supremum of our sets $\Delta(P)$ could be related to the several attempts to quantify the fatness of a polygon (see [4]) but our approach is different: we look at the whole set $\Delta(P)$, not only at its supremum.

If $P$ is convex and $\alpha(P)>\pi / 2$ then $\Delta(P)=(0, \mu(P)]$, where $\mu(P)$ is the length of $P$ 's shortest side. In this particular case the arguments below could be rephrased in terms of $\mu(P)$. However, in [1] we care mainly about rectangles and if $R$ is a rectangle then $\Delta(R)=(0, \mu(R))$ does not contain $\mu(R)$. Therefore, in order to unify the treatment of rectangles and polygons with bigger internal angles we chose to use $\Delta(P)$.

Using $\alpha(P)$ and $\Delta(P)$ we can state the key results behind most arguments in this paper. Given a family $\mathcal{P}$ of polygons and a function $S: \mathcal{P} \rightarrow \operatorname{Sub}\left(\mathbb{R}^{2}\right)$, Theorems 1 and 2 give sufficient conditions for $S$ to be a sentinel assignment for $\mathcal{P}$ with respect to $I_{2}$. Lemmas 1,2 and 3 present technical results used to prove the theorems.

Lemma 1. Let $P$ and $Q$ be polygons with $\alpha(P) \geq \pi / 2$ and $\alpha(Q) \geq \pi / 2$. Suppose $u, v, w \in q_{k} q_{k+1}$ are such that $\left\|u-q_{k}\right\|<\left\|v-q_{k}\right\|<\left\|w-q_{k}\right\|$ and
(a) $u, w \notin \operatorname{int}(P)$,
(b) $v \in \operatorname{int}(P)$ and
(c) $\|u-w\| \in \Delta(P) \cap \Delta(Q)$.

If no vertex of $P$ is in the interior of $Q$ then there exists a vertex $p_{j}$ of $P$ with $p_{j-1} p_{j} \cap u v=\{x\}$ and $p_{j} p_{j+1} \cap v w=\{y\}$ (see Fig. 4). Moreover, $x$ and $y$ are such that $x y-\{x, y\} \subset \operatorname{int}(P)$,

$$
\left\|p_{j}-x\right\|<\|u-w\| \quad \text { and } \quad\left\|p_{j}-y\right\|<\|u-w\| .
$$
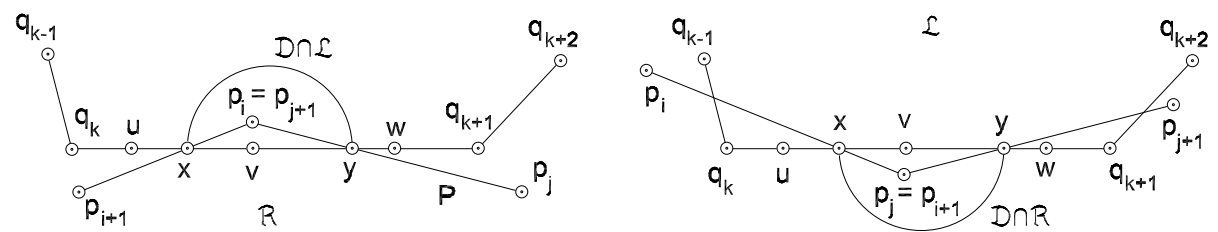

Figure 4 - The conceivable situations for $p \in p_{i} p_{i+1} \cap p_{j} p_{j+1}$. Actually, only the right one is valid. 
Proof of Lemma 1. Let $x y$ be the biggest segment contained in $q_{k} q_{k+1}$ such that $v \in x y$ and $x y-\{x, y\} \subset \operatorname{int}(P)$ and consider the sides $p_{i} p_{i+1}$ and $p_{j} p_{j+1}$ such that $x \in p_{i} p_{i+1}$ and $y \in p_{j} p_{j+1}$. Items (a) and (b) imply that $x y \subset u w$. Item (c) shows that $\|x-y\| \leq\|u-w\| \in \Delta(P)$. Since $x \in p_{i} p_{i+1}$ and $y \in p_{j} p_{j+1}$, the definition of $\Delta$ implies that $p_{i} p_{i+1} \cap p_{j} p_{j+1} \neq \varnothing$. Let $p \in\left\{p_{j}, p_{j+1}\right\}$ be the common vertex of $p_{i} p_{i+1}$ and $p_{j} p_{j+1}$. According to Figure 3, $p$ belongs to the disk $\mathcal{D}=\left\{z \in \mathbb{R}^{2}\right.$ with $\left.\|2 z-(x+y)\| \leq\|x-y\|\right\}$. If $\mathcal{L}$ and $\mathcal{R}$ are the open half planes on the left and right sides of the line $q_{k} q_{k+1}$ then, in principle, we have the two possibilities regarding $p$ described in Figure 4. However, the situation on the left side of this figure does not occur, because $\mathcal{D} \cap \mathcal{L} \subset \operatorname{int}(Q)$ and there is no vertex of $P \operatorname{in} \operatorname{int}(Q)$ by hypothesis. In fact, notice that $\mathcal{D} \cap \mathcal{L}$ cannot intersect sides of $Q$ which are nonconsecutive to $q_{k} q_{k+1}$ because (c) implies that the distances from such sides to $q_{k} q_{k+1}$ is bigger than the radius of $\mathcal{D}$. Moreover, $\mathcal{D} \cap \mathcal{L}$ does not intersect the sides $q_{k-1} q_{k}$ and $q_{k+1} q_{k+2}$ either, because the internal angles of $Q$ are at least $\pi / 2$. This implies that $\mathcal{D} \cap \mathcal{L}$ is contained in the interior of $Q$ and, thus, $p \notin \mathcal{D} \cap \mathcal{L}$ as we claimed. Therefore, we must have $p=p_{j}=p_{i+1} \in \mathcal{D} \cap \mathcal{R}$ as described in the right side of Figure 4 and the bounds in (2) holds because the diameter of $\mathcal{D}$ is at most $\|u-w\|$. (For further reference, as $p_{i+1} \equiv p_{j}$, we will refer to $p_{i} p_{i+1}$ as $p_{j-1} p_{j}$.)

Lemma 2. Let $P, Q$ and $\{x\}=q_{k} q_{k+1} \cap p_{j-1} p_{j}$ as in Lemma 1. Assume that $x \neq q_{k}$ and let $c \in \operatorname{border}(P) \cap \operatorname{border}(Q)$ be the first point different from $x$ encountered when walking $x p_{j-1}$ from $x$ to $p_{j-1}$ (see Fig. 5$)^{1}$. Then, either

$$
c \in q_{k-1} q_{k}-\left\{q_{k}\right\} \text { and }\|x-c\|>\max \left\{\left\|c-q_{k}\right\|,\left\|q_{k}-x\right\|\right\} \text {, }
$$

or

$$
c \notin q_{k-1} q_{k} \cup q_{k} q_{k+1} \text { and }\|x-c\| \geq \sup \Delta(Q) \text {. }
$$

Proof of Lemma 2. Let $q_{m} q_{m+1}$ be the side of $Q$ containing $c$. We will first show that $q_{m} q_{m+1} \neq q_{k} q_{k+1}$ and $q_{m} q_{m+1} \neq q_{k+1} q_{k+2}$. Since $x \in q_{k} q_{k+1}-$

\footnotetext{
${ }^{1}$ Note that $p_{j-1} p_{j} \cap u v=p_{j-1} p_{j} \cap q_{k} q_{k+1}=\{x\} \neq q_{k}$ implies that the segments $q_{k} q_{k+1}$ and $p_{j-1} p_{j}$ are not colinear and the point $c$ is well defined.
} 


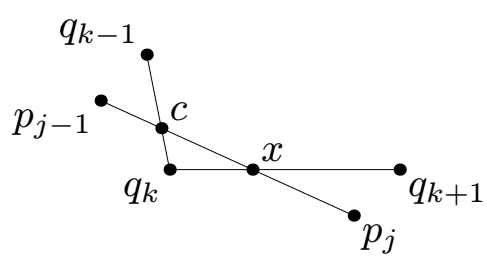

(i)

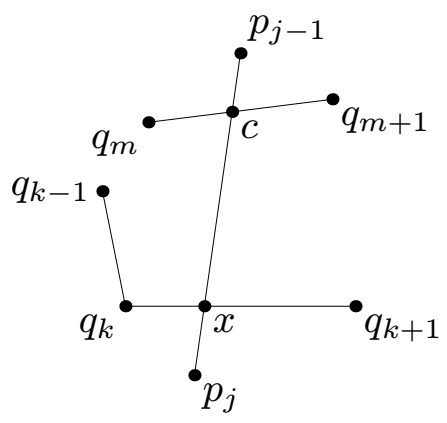

(ii)

Figure 5 - The situations (i) and (ii) described in Lemma 2.

$\left\{q_{k}\right\}, c \in q_{m} q_{m+1}$ and $\emptyset \neq x c-\{x, c\} \subset \operatorname{int}(Q)$ we have that $x$ and $c$ are in different sides of $Q$ and, in consequence, $q_{m} q_{m+1} \neq q_{k} q_{k+1}$. Considering the triangle with vertices $x p_{j} y$ (right hand side of Fig. 4) and knowing that $\angle x p_{j} y=\angle p_{j-1} p_{j} p_{j+1} \geq \pi / 2$ we have that $\angle q_{k} x c \cong \angle p_{j} x y<\pi / 2$. Then $\angle q_{k} x c<\pi / 2$ implies that $\angle q_{k+1} x c>\pi / 2$. This and $\angle q_{k} q_{k+1} q_{k+2} \geq \pi / 2$ imply that the half lines $x+\gamma_{1}(c-x)$ and $q_{k+1}+\gamma_{2}\left(q_{k+2}-q_{k+1}\right), \gamma_{1}, \gamma_{2} \geq 0$, never intersect. Thus, $c \notin q_{k+1} q_{k+2}$ and $q_{m} q_{m+1} \neq q_{k+1} q_{k+2}$.

Therefore, we have only these two possibilities regarding $q_{m} q_{m+1}$ :

- $q_{m} q_{m+1}=q_{k-1} q_{k}$. In this case $\|x-c\|>\max \left\{\left\|c-q_{k}\right\|,\left\|q_{k}-x\right\|\right\}$ because $x c$ is the biggest side in the (nonempty) triangle with vertices $x q_{k} c$, since the angle $\angle x q_{k} c$ is equal to $\angle q_{k+1} q_{k} q_{k-1}$, which is at least $\pi / 2$.

- $q_{m} q_{m+1} \neq q_{k-1} q_{k}$. In this case, by definition of $\Delta,\|x-c\|>\delta$ for all $\delta \in \Delta(Q)$. This implies that $\|x-c\| \geq \sup \Delta(Q)$.

Clearly, a symmetric result can be obtained for $\{y\}=q_{k-1} q_{k} \cap p_{j} p_{j+1}$ defined in Lemma 1.

Part of the hypothesis of the main theorem in this section requires that each polygon in $\mathcal{P}$ has an internal sentinel outside the forbidden region $F(P, \delta)$ that we now describe. Suppose $P$ is a polygon and $\delta>0$ satisfies $\delta \leq\left\|p_{i+1}-p_{i}\right\|$ for all $i$. For each vertex $p_{i}$ of $P$ we define the forbidden corner $F C(P, i, \delta)$ as $F C(P, i, \delta)=\left\{x=\lambda u+(1-\lambda) v\right.$ for $\lambda \in(0,1), u \in p_{i-1} p_{i}, v \in p_{i} p_{i+1}$,
$u v-\{u, v\} \subset \operatorname{int}(P)$ and $\|u-v\|<\delta\}$ 
(see Fig. 6) and we define the forbidden region

$$
F(P, \delta)=\bigcup_{i: \angle p_{i-1} p_{i} p_{i+1}<\pi} F C(P, i, \delta) .
$$

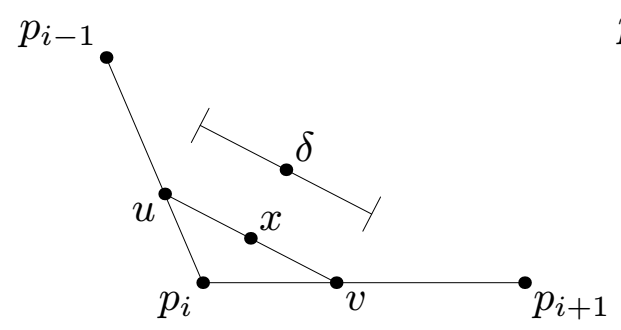

(a)

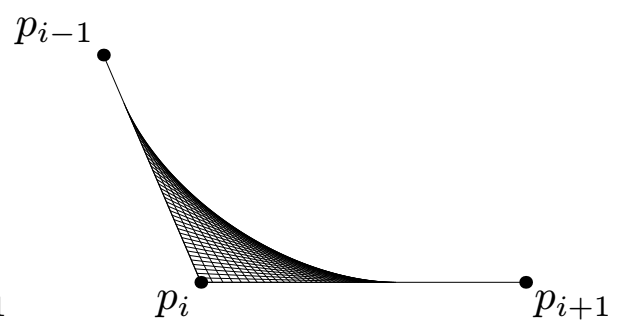

(b)

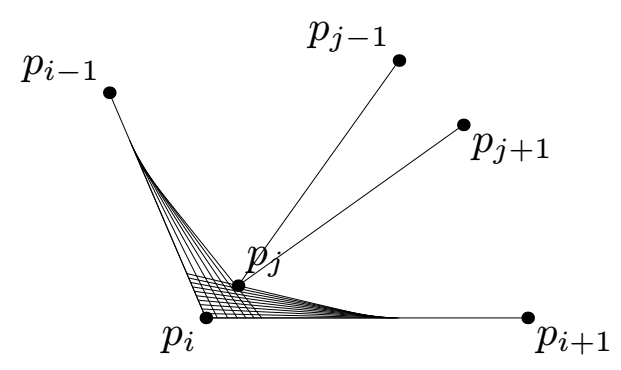

(c)

Figure 6 - The forbidden corner $F C(P, i, \delta)$.

The next lemma shows that we can always find internal sentinels outside the forbidden regions for any polygon with $n>3$ vertices.

Lemma 3. If $P$ is a polygon with $n>3$ vertices and $\delta \in \Delta(P)$ then the set $\operatorname{int}(P)-F(P, \delta)$ is not empty.

Proof of Lemma 3. We analyze convex and non-convex polygons separately.

Let us start with $P$ convex. For each $i=0, \ldots, n-1$ let $P_{i}$ be the convex polygon with vertices $\left\{p_{0}, \ldots, p_{n-1}\right\}-\left\{p_{i}\right\}$. Note that

$$
P_{i} \cap P_{j} \cap P_{k} \supset\left\{p_{0}, \ldots, p_{n-1}\right\}-\left\{p_{i}, p_{j}, p_{k}\right\} \neq \emptyset
$$


for every triplet $i, j, k$. Therefore, Radon's theorem implies that

$$
A=\bigcap_{i=0}^{n-1} P_{i} \neq \emptyset .
$$

The set $A$ does not contain vertices or sides of $P$. Thus, $A \subset \operatorname{int}(P)$. Moreover, the interior of the ears of $P$ do not intersect $A$. It is clear then that $\operatorname{int}(P)-$ $F(P, \delta) \supset A \neq \emptyset$ and we are done with the convex case.

If $P$ is not convex then there exists at least one $i$ such that the angle $\angle p_{i-1} p_{i} p_{i+1}$ is bigger than $\pi$. Let $\epsilon \in(0, \delta / 4)$ be such that

$$
s=s_{\epsilon}=p_{i}-\epsilon\left(\frac{p_{i+1}-p_{i}}{\left\|p_{i+1}-p_{i}\right\|}+\frac{p_{i-1}-p_{i}}{\left\|p_{i-1}-p_{i}\right\|}\right)
$$

belongs to int $(P)$. Geometrically (see Fig. 7), $s$ is a point in the bisectrix of the angle $\angle p_{i-1} p_{i} p_{i+1}$ very close to $p_{i}$ :

$$
\left\|p_{i}-s\right\| \leq \epsilon\left\|\frac{p_{i+1}-p_{i}}{\left\|p_{i+1}-p_{i}\right\|}+\frac{p_{i-1}-p_{i}}{\left\|p_{i-1}-p_{i}\right\|}\right\| \leq 2 \epsilon<\delta / 2 .
$$

To complete this proof we will show that $s \in \operatorname{int}(P)-F(P, \delta)$, i.e., it does not belong to any forbidden corner $F C(P, j, \delta)$ with $\angle p_{j-1} p_{j} p_{j+1}<\pi$. Consider a forbidden corner $F C(P, j, \delta)$ as above. Let $u \in p_{j-1} p_{j}$ and $v \in p_{j} p_{j+1}$ be such that $s \in u v, u v-\{u, v\} \in \operatorname{int}(P)$. To prove that $s \notin F C(P, j, \delta)$ is enough to show that $\|u-v\| \geq \delta$. Noticing that $\angle p_{j-1} p_{j} p_{j+1}<\pi<\angle p_{i-1} p_{i} p_{i+1}$ implies $i \neq j$, we are left with the three cases:

(i) $j=i+1$ (described in Fig. 7a),

(ii) $j=i-1$ (described, after reflection, in Fig. 7a),

(iii) $j \notin\{i-1, i, i+1\}$ (described in Fig. 7b).

Cases (i) and (ii) differ only by a reflection and we will threat both as case (i).

Let us start with case (i). Since $P$ has more than three vertices, we have that $p_{i-1} p_{i}$ and $p_{i+1} p_{i+2}$ are not consecutive. Thus, by definition of $\Delta(P)$ we have that $\left\|p_{i}-v\right\|>\delta$. Now,

$$
\angle v p_{i} u>\angle s p_{i} u=\angle p_{i-1} p_{i} p_{i+1} / 2>\pi / 2 \text {. }
$$




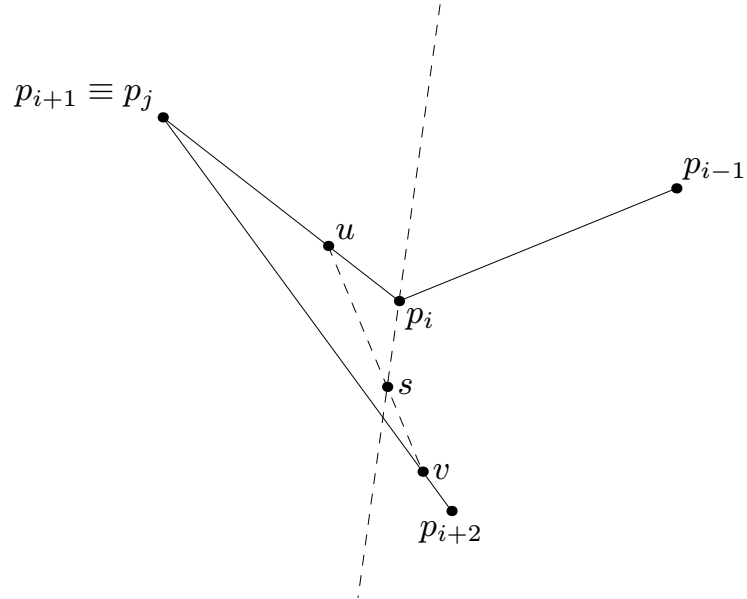

(a)

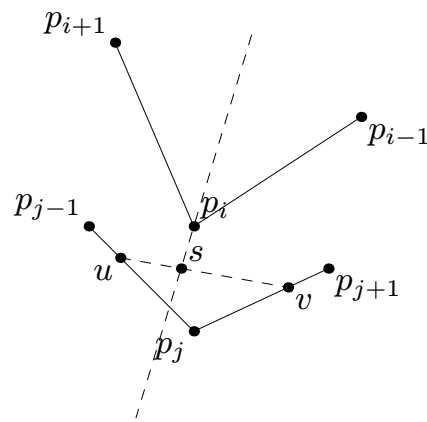

(b)

Figure 7 - The two cases that illustrates the proof of Lemma 3.

This implies that $\|u-v\|>\left\|p_{i}-v\right\|>\delta$ and we are done with cases (i) and (ii).

Finally, let us handle case (iii). In this case the sides $p_{i} p_{i+1}$ and $p_{j} p_{j+1}$ are not consecutive. Thus, by definition of $\delta,\left\|p_{i}-v\right\|>\delta$. As a consequence,

$$
\|v-s\| \geq\left\|p_{i}-v\right\|-\left\|s-p_{i}\right\|>\delta-\delta / 2=\delta / 2 .
$$

Analogously, since the sides $p_{i-1} p_{i}$ and $p_{j-1} p_{j}$ are not consecutive we get that $\left\|p_{i}-u\right\|>\delta$ and then

$$
\|s-u\| \geq\left\|p_{i}-u\right\|-\left\|s-p_{i}\right\|>\delta-\delta / 2=\delta / 2 .
$$

Combining (6) and (7) we get

$$
\|u-v\|=\|u-s\|+\|s-v\|>\delta / 2+\delta / 2=\delta
$$

and the proof is complete.

Now we can address the main question of this section: Given a family $\mathcal{P}$ of polygons and a function $S: \mathcal{P} \rightarrow \operatorname{Sub}\left(\mathbb{R}^{2}\right)$, give sufficient conditions for $S$ to be a sentinel assignment for $\mathcal{P}$ with respect to $I_{2}$. 
Theorem 1. Let $\mathcal{P}$ be a family of polygons such that, for each $P \in \mathcal{P}, \alpha(P) \geq$ $\pi / 2$. Let $S$ be a function from $\mathcal{P}$ to $\operatorname{Sub}\left(\mathbb{R}^{2}\right)$. Assume that for all $P \in \mathcal{P}$

(a) $S(P)$ contains the vertices of $P$;

and that there exists $\delta \in \bigcap_{P \in \mathcal{P}} \Delta(P)$ for which

(b) for each side $p_{i} p_{i+1}$ of $P$ there exist $n_{i} \in \mathbb{N}$ and $s_{j} \equiv p_{i}+\left(\gamma_{j} / \Gamma\right)$ $\left(p_{i+1}-p_{i}\right) \in S(P)$, where $\Gamma=\left\|p_{i+1}-p_{i}\right\|$, such that $\gamma_{j} \in[0, \Gamma]$ for $j=1, \ldots, n_{i}$, if $n_{i} \neq 0$ then $\gamma_{1}=\delta$ and $\gamma_{n_{i}}=\Gamma-\delta, \gamma_{j}<\gamma_{j+1}$ and $\left|\gamma_{j}-\gamma_{j+1}\right| \leq \delta$ for $j=1, \ldots, n_{i}-1$;

(c) the set $S(P)$ contains a point in $\operatorname{int}(P)-F(P, \delta)$.

Then $S$ is a sentinel assignment for $\mathcal{P}$ with respect to $I_{2}$.

Proof of Theorem 1. Consider members $A$ and $B$ of $\mathcal{P}$ and $T, U \in I_{2}$. Define $P=T(A), Q=U(B), \bar{S}(P)=T(S(A))$ and $\bar{S}(Q)=U(S(B))$. Since $T$ and $U$ are isometries, hypotheses (a), (b) and (c) also apply to $P$ and $\bar{S}(P)$ and $Q$ and $\bar{S}(Q)$. To prove Theorem 1, we assume that there exists a point $z \in \operatorname{int}(P) \cap \operatorname{int}(Q)$ and show that either

$$
\bar{S}(P) \cap \operatorname{int}(Q) \neq \emptyset \quad \text { or } \quad \bar{S}(Q) \cap \operatorname{int}(P) \neq \emptyset .
$$

Hypothesis (c) implies that there exists $s \in \bar{S}(P) \cap(\operatorname{int}(P)-F(P, \delta))$. If $s \in \operatorname{int}(Q)$ then the first condition in (8) is satisfied and we are done. Thus, we assume that $s \notin \operatorname{int}(Q)$. Since $s$ and $z$ are in the interior of the Jordan curve $P$, there exists a continuous path contained in the interior of $P$ connecting $S$ to $z$. This path intersects border $(Q)$ at some point $v$ because $s \notin \operatorname{int}(Q)$ and $z \in \operatorname{int}(Q)$. If $v \in \bar{S}(Q)$ then the second condition in (8) is satisfied and we are done again. Thus, we only need to care about the case in which $v \notin \bar{S}(Q)$ and $v$ is the first intersection of the path above and $\operatorname{border}(Q)$, that is, there exists a continuous function $\phi:[0,1] \rightarrow \mathbb{R}^{2}$ such that

$$
\phi(0)=s, \quad \phi(1)=v, \quad \phi([0,1]) \subset \operatorname{int}(P) \quad \text { and } \quad \phi([0,1)) \cap Q=\emptyset .
$$

If $q_{k} q_{k+1}$ is the side of $Q$ that contains $v$, then by hypotheses (a) and (b) there exist $u, w \in \bar{S}(Q) \cap q_{k} q_{k+1}$ such that $v \in u w,\|u-w\| \leq \delta$ and $\left\|u-q_{k}\right\|<$ 


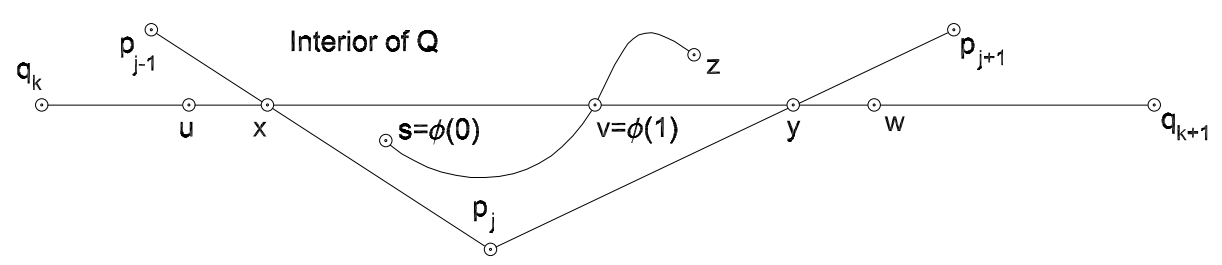

Figure 8 - The relative position of $p_{j}, s, u, v$ and $w$ when $\{u, w\} \cap \operatorname{int}(P)=\emptyset$.

$\left\|w-q_{k}\right\|$. If $u$ or $w$ belong to $\operatorname{int}(P)$ then the second condition in (8) is satisfied and we are done. To complete this proof, let us now derive a contradiction from the assumption that hypothesis (c) holds and neither $u$ nor $w$ belong to $\operatorname{int}(P)$. In this case Lemma 1 implies that there exist $p_{j-1}, p_{j}$ and $p_{j+1}$, vertices of $P$, and $\{x\}=u v \cap p_{j-1} p_{j},\{y\}=v w \cap p_{j} p_{j+1}$ and $x y-\{x, y\} \subset \operatorname{int}(P)$ like the ones in Figure 8.

This figure is also accurate regarding the fact that $s$ is in the interior of the triangle $\tau=x p_{j} y$. In fact, (9) implies that the path $\phi([0,1])$ does not cross $x p_{j}$ nor $p_{j} y$ and $\phi([0,1))$ does not touch $y x$. Moreover, if $\epsilon$ is small $\phi(1-\epsilon)$ is close to $v$ and outside $Q$. This implies that $\phi(1-\epsilon) \in \operatorname{int}(\tau)$ for $\epsilon$ small. Since $\phi([0,1)]$ does not touch $\operatorname{border}(\tau)$ we have that $\phi([0,1)) \subset \operatorname{int}(\tau)$. In particular, $s=\phi(0) \in \operatorname{int}(\tau)$. However, equation (2) shows that $\left\|x-p_{j}\right\| \leq \delta$ and $\left\|p_{j}-y\right\| \leq \delta$. This implies that $\operatorname{int}(\tau) \subset F C(P, j, \delta)$ and we deduce that $s \in F(P, \delta)$. This conclusion contradicts our choice of $s$ at the beginning of this proof.

Theorem 1 implies that if $\mathcal{P}$ is a finite family of polygons $P$ with $\alpha(P) \geq \pi / 2$ then there exists a sentinel assignment $S$ for $\mathcal{P}$ with respect to $I_{2}$ such that $S(P)$ is finite for all $P \in \mathcal{P}$ : take an arbitrary choice of $\delta \in \bigcap_{P \in \mathcal{P}} \Delta(P)$, for example,

$$
\delta=\frac{1}{3} \sup \bigcap_{P \in \mathcal{P}} \Delta(P)>0,
$$

and for each $P \in \mathcal{P}$ choose a finite set $S(P)$ of sentinels that satisfy the conditions (a)-(c) in Theorem 1. Lemma 3 guarantees that it is possible to find a point satisfying condition (c) of Theorem 1 .

Using Theorem 1 you can prove the following corollary to justify our claim regarding the intersections of rotated and translated copies of the pentagon and the hexagon in the first page of this paper: 
Corollary 1. Let $\delta>0$ and let $\mathcal{P}$ be a family of convex polygons such that, for all $P \in \mathcal{P}$ we have that $\alpha(P)>\pi / 2$ and all sides of $P$ have length $\delta$. For $P \in \mathcal{P}$ take $s \in \operatorname{int}(P)-F(P, \delta)$ and define $S(P)=\left\{s, p_{1}, p_{2}, \ldots, p_{n(P)}\right\}$. The function $S$ is a sentinel assignment for $\mathcal{P}$ regarding $I_{2}$. (S satisfies hypotheses (a) and (c) by definition and hypothesis $(b)$ with $n_{i}=0$.)

We have seen that Theorem 1 can be used to construct sentinel assignments for certain families of polygons or to verify that a given assignment $S$ that satisfies the hypotheses of Theorem 1 is a sentinel assignment. However, there is a simple and important case that is not covered by Theorem 1. Consider a family $\mathcal{P}$ of identical squares $P$ of side $d$. In this case we have that $\Delta(P)=(0, d)$ for all $P \in \mathcal{P}$. Let $\delta=\frac{1}{2} d$. Consider a sentinel assignment $S$ for $\mathcal{P}$ such that, for each $P \in \mathcal{P}, S(P)$ is given by a sentinel in each vertex of $P$, a sentinel in the middle of each side of $P$ and a sentinel in $\operatorname{int}(P)-F(P, \delta)$. This $S$ satisfies hypotheses (a) and (b) but does not satisfy hypothesis (c) of Theorem 1. Therefore, Theorem 1 can not be used to certify that $S$ is, in fact, a sentinel assignment for $\mathcal{P}$. The theorem below is similar to Theorem 1 except for the fact that hypothesis (c) is replaced by a couple of other hypotheses satisfied by the sentinel assignment described above.

Theorem 2. Let $\mathcal{P}$ be a family of polygons such that, for all $P \in \mathcal{P}, \alpha(P) \geq$ $\pi / 2$. Let $S$ be a function from $\mathcal{P}$ to $\operatorname{Sub}\left(\mathbb{R}^{2}\right)$. Assume that for all $P \in \mathcal{P}$

(a) $S(P)$ contains the vertices of $P$;

and that there exists $\delta \in \bigcap_{P \in \mathcal{P}} \Delta(P)$ that satisfies

(b) for each side $p_{i} p_{i+1}$ of $P$ there exist $n_{i} \in \mathbb{N}$ and $s_{j} \equiv p_{i}+\left(\gamma_{j} / \Gamma\right)\left(p_{i+1}-\right.$ $\left.p_{i}\right) \in S(P)$, where $\Gamma=\left\|p_{i+1}-p_{i}\right\|$, such that $\gamma_{j} \in[0, \Gamma]$ for $j=$ $1, \ldots, n_{i}$, if $n_{i} \neq 0$ then $\gamma_{1}=\delta$ and $\gamma_{n_{i}}=\Gamma-\delta, \gamma_{j}<\gamma_{j+1}$ and $\left|\gamma_{j}-\gamma_{j+1}\right| \leq \delta$ for $j=1, \ldots, n_{i}-1$;

(c) $S(P) \cap \operatorname{int}(P) \neq \emptyset$; and

(d) $\left\|p_{i+1}-p_{i}\right\| \geq 2 \delta$ for all $i \in \mathbb{Z}$.

Then $S$ is a sentinel assignment for $\mathcal{P}$ with respect to $I_{2}$. 
Proof of Theorem 2. This proof follows very closely the proof of Theorem 1 . Thus, we will assume that there exists a point $z \in \operatorname{int}(P) \cap \operatorname{int}(Q)$ and show that either

$$
\bar{S}(P) \cap \operatorname{int}(Q) \neq \emptyset \quad \text { or } \quad \bar{S}(Q) \cap \operatorname{int}(P) \neq \emptyset .
$$

By hypothesis (c), there exists $s \in \bar{S}(P) \cap \operatorname{int}(P)$ and let $v \in \operatorname{int}(P)$ be the first point that belongs to border $(Q)$ encountered when walking the continuous path from $s$ to $z$ trought the interior of $P$. If $s \in \operatorname{int}(Q)$ or if $v \in \bar{S}(Q)$ then (10) holds and we are done. Therefore, we only need to care about the case in which there exists a continuous function $\phi:[0,1] \rightarrow \mathbb{R}^{2}$ such that

$$
\phi(0)=s, \quad \phi(1)=v, \quad \phi([0,1]) \subset \operatorname{int}(P) \text { and } \phi([0,1)) \cap Q=\emptyset .
$$

Let $q_{k} q_{k+1}$ be the side of $Q$ that contains $v$. Then, hypotheses (a) and (b) imply that there exist $u, w \in \bar{S}(Q) \cap q_{k} q_{k+1}$ such that $v \in u w,\|u-w\| \leq \delta$ and $\left\|u-q_{k}\right\|<\left\|w-q_{k}\right\|$. If $\bar{S}(Q) \cap \operatorname{int}(P) \neq \emptyset$ we are done. So, we assume that $u, w \notin \operatorname{int}(P)$. In this case Lemma 1 implies that there exist $p_{j-1}, p_{j}$ and $p_{j+1}$, vertices of $P$, and $\{x\}=u v \cap p_{j-1} p_{j}$ and $\{y\}=v w \cap p_{j} p_{j+1}$ like the ones in right hand side of Figure 4. Hypothesis (d) and the fact that $\|u-w\| \leq \delta$ imply that either $u \neq q_{k}$ or $w \neq q_{k+1}$. By symmetry, assume that $u \neq q_{k}$ which implies that $x \neq q_{k}$ (in fact, it implies that $\left\|x-q_{k}\right\| \geq$ $\delta)$. In this case, by Lemma 2, there exists $c \in \operatorname{border}(P) \cap \operatorname{border}(Q)$, the first point different from $x$ encountered when walking $x p_{j-1}$ from $x$ to $p_{j-1}$ (see Fig. 5), such that either (3) or (4) holds. By (3) and the fact that $\| x-$ $q_{k} \| \geq \delta$ or by (4) and the fact that $\delta \in \Delta(Q)$, we have that $\|x-c\| \geq \delta$. So, we have $x c \subset \operatorname{int}(Q) \cap p_{j-1} p_{j}$ and $\|x-c\| \geq \delta$. By hypothesis (b) there exists $t \in x c \cap \bar{S}(P)$ and, thus, we have verified (10).

\section{Sentinels for rectangles}

In this section we present optimal sentinel assignments for rectangles with respect to $I_{2}^{+}$. We assume that $a=a(R)=\left\|r_{0}-r_{1}\right\|$ is the longest side of the rectangle $R$ and $b=b(R)=\left\|r_{0}-r_{3}\right\|$ is the smallest side. We define sentinel assignments that depend on two parameters $\delta$ and $\rho$ : given a rectangle $R$ we define $S_{\delta \rho}(R)$ as the set of points indicated by circles in Figure 9. 

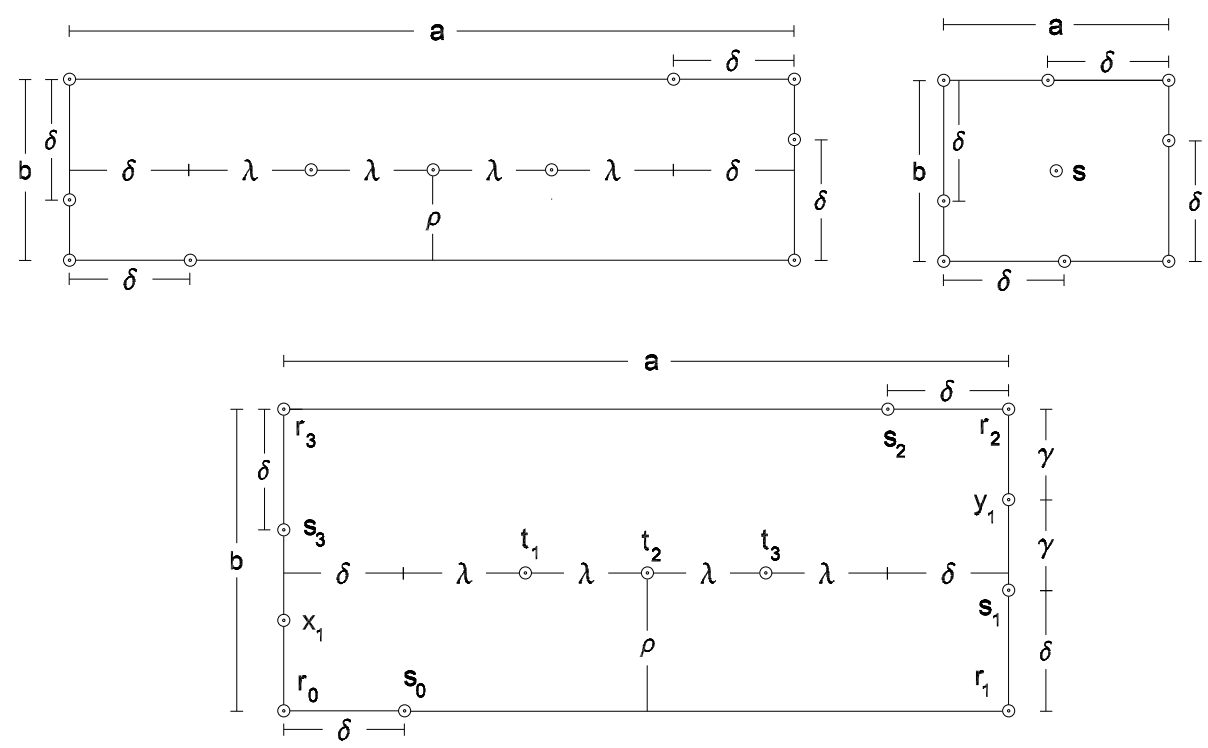

Figure 9 - The sentinels $S_{\delta \rho}(R)$. In the top left rectangle $a>2 \delta \geq b>\delta$ and $\lambda=(a-2 \delta) / n_{\lambda}$ for $n_{\lambda}=\lceil(a-2 \delta) / \delta\rceil$. For the bottom rectangle, $b>2 \delta$ and $\gamma=(b-\delta) / n_{\gamma}$ with $n_{\gamma}=\lceil(b-\delta) / \delta\rceil$. In the top right rectangle $a \leq 2 \delta$ and $b \leq 2 \delta$.

Formally, given a rectangle $R$ and $\delta$ and $\rho$ with $0<\delta, \rho<\left\|r_{3}-r_{0}\right\|$ we define $S_{\delta \rho}(R)$ as the set formed by:

1. The vertices $r_{0}, r_{1}, r_{2}, r_{3}$ of $R$.

2. The points $s_{i}=r_{i}+\delta\left(r_{i+1}-r_{i}\right) /\left\|r_{i+1}-r_{i}\right\|$ for $i=0,1,2$ and 3 .

3. If $b>2 \delta$, the points $x_{i}=s_{3}+i\left(r_{0}-s_{3}\right) / n_{\gamma}$ and $y_{i}=s_{1}+i\left(r_{2}-s_{1}\right) / n_{\gamma}$ for $1 \leq i<n_{\gamma}$, with $n_{\gamma}=\lceil(b-\delta) / \delta\rceil$.

4. If $a>2 \delta$, the points $t_{i}=\rho\left(r_{3}-r_{0}\right) / b+\left(s_{0}-r_{0}\right)+i \Delta / n_{\tau}$, for $1 \leq i<n_{\tau}$, with $\Delta=r_{1}-r_{0}-2\left(s_{0}-r_{0}\right)$ and $n_{\tau}=\lceil\|\Delta\| / \delta\rceil$.

5. If $a \leq 2 \delta$, the point $s=\left(r_{0}+r_{1}+r_{2}+r_{3}\right) / 4$.

The following theorem is the main result in this section:

Theorem 3. Let $\mathcal{R}$ be a family of rectangles and suppose $\delta \in \bigcap_{R \in \mathcal{R}} \Delta(R)$. If for all $R \in \mathcal{R}$ we have $\rho(R) \in(0, b(R))$ such that

$$
b\left(R^{\prime}\right) \notin\{\rho(R), b(R)-\rho(R)\} \quad \text { for all } \quad R^{\prime} \in \mathcal{R}
$$


then the function $S: \mathcal{R} \rightarrow \operatorname{Sub}\left(\mathbb{R}^{2}\right)$ given by $S(R)=S_{\delta \rho(R)}(R)$ is a sentinel assignment for $\mathcal{R}$ with respect to $I_{2}^{+}$.

If all rectangles in $R \in \mathcal{R}$ have $\delta<b(R) \leq 2 \delta$ then the sets $S_{\delta \rho(R)}(R)$ contain only one element in each smaller side of $R$ and the sentinel assignment $S$ above in Theorem 3 is optimal, i.e., if $S^{\prime}$ is another sentinel assignment for $\mathcal{R}$ with respect to $I_{2}^{+}$then $S^{\prime}(R)$ has at least as many elements as $S(R)$. In fact, $S^{\prime}(R)$ must contain the vertices of $R$ and at least one element in each side of $R$. Moreover, if $S^{\prime}(R)$ does not contain as many elements as $S(R)$ then it is possible to superimpose a copy of $R$ rotated by $\pi / 2$ and $R$ in order to contradict the definition of sentinel assignment.

We end this paper with the proof of Theorem 3:

Proof of Theorem 3. The sets $S_{\delta \rho}(R)$ and the condition (12) are invariant under $I_{2}^{+}$, in the sense that if $T \in I_{2}^{+}$and $R \in \mathcal{R}$ then $S_{\delta \rho}(T(R))=T\left(S_{\delta \rho}(R)\right)$ and $b(R)=b(T(R))$. Therefore, to prove Theorem 3 it is enough to show that if $H$ and $R$ are rectangles with $\left\|h_{0}-h_{3}\right\| \geq\left\|h_{1}-h_{2}\right\|,\left\|r_{0}-r_{3}\right\| \geq\left\|r_{1}-r_{2}\right\|$ and

$$
\delta \in \Delta(H) \cap \Delta(R), \quad\left|\left\|r_{0}-r_{3}\right\|-\left\|h_{0}-h_{3}\right\|\right| \notin\{\rho(H), \rho(R)\}
$$

and $\operatorname{int}(H) \cap \operatorname{int}(R) \neq \emptyset$ then

$$
\operatorname{int}(H) \cap S_{\delta \rho(R)}(R) \neq \emptyset \quad \text { or } \quad S_{\delta \rho(H)}(H) \cap \operatorname{int}(R) \neq \emptyset .
$$

If $a(H) \leq 2 \delta$ and $a(R) \leq 2 \delta$ then (14) is a consequence of Theorem 1 applied to $\mathcal{P}=\{H, R\}$. Thus, we can assume that $a(H)=\left\|h_{1}-h_{0}\right\|>2 \delta$. Moreover, if $s_{i}$ are the sentinels described in Figure 9 for $H$ and $\left\{h_{0}, h_{1}, h_{2}, h_{3}\right.$, $\left.s_{0}, s_{1}, s_{2}, s_{3}\right\} \cap \operatorname{int}(R) \neq \varnothing$ then (14) is satisfied. Therefore, we only need to analyze the case

$$
a(H)=\max \{a(H), a(R)\}>2 \delta \text { and } h_{i}, s_{i} \notin \operatorname{int}(R) \text { for } i=0,1,2,3 .
$$

We leave to the reader, the verification of the fact that if $h_{0} h_{1}$ and $r_{0} r_{1}$ are parallel then (14) is satisfied ${ }^{2}$. From now on we analyze the case in which $H$ is horizontal and $R$ is a rotated rectangle as in Figure 10 or a rotated rectangle with $a(R) \leq 2 \delta$.

\footnotetext{
2 when doing that, do not forget hypothesis (12).
} 

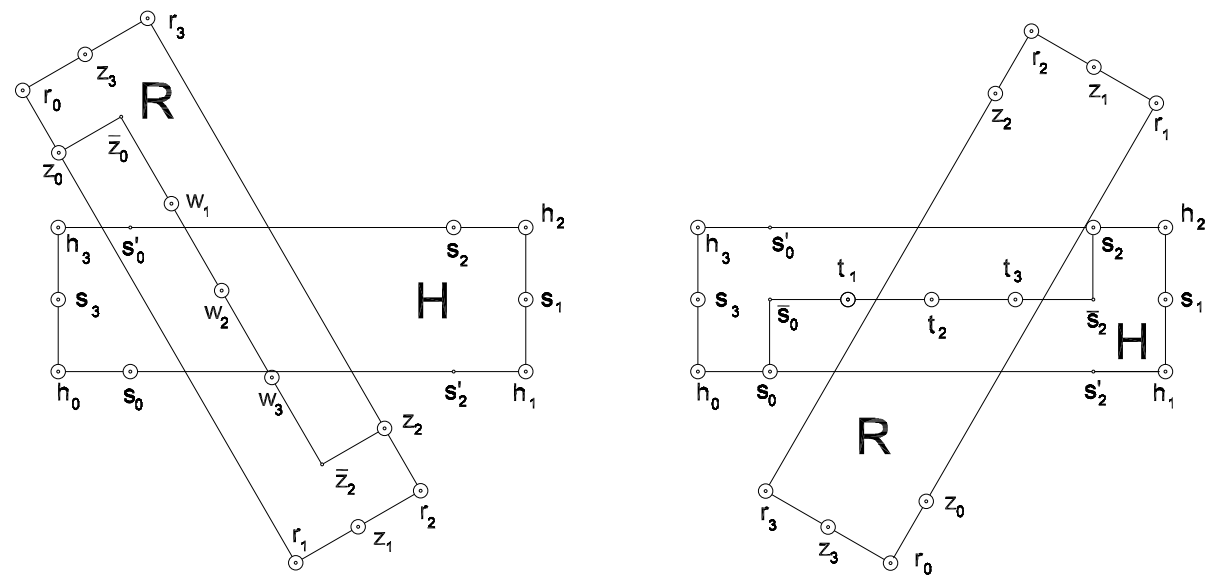

Figure 10 - Two ways a rotated rectangle $R$ could cross the horizontal rectangle $\mathrm{H}$ and do not touch $h_{3} h_{0} \cup h_{0} s_{0} \cup h_{1} h_{2} \cup h_{2} s_{2}$. Sentinels are indicated by large circles and auxiliary points have names with superscripts and are represented by small circles.

To avoid conflicting names, we rename $R$ 's sentinels as in Figure 10: the $s$ sentinels for $R$ are called $z$ 's and the $t$ sentinels are called $w$. Notice that in all polygons $R$ in Figure 9 if $r_{i}$ is a vertex of $R$ then there exists a sentinel $z_{i}$ at a distance $\delta$ from $r_{i}$ in the counterclockwise direction along $R$. Repeating the argument in the second paragraph of the proof of Theorem 1 with $u=h_{0}$, $w=s_{0}$ and $q_{k+1}=h_{1}$, we deduce that if $\operatorname{int}(R) \cap h_{0} s_{0} \neq \emptyset$ then $\left\{z_{0}, z_{1}, z_{2}, z_{3}\right\} \cap$ $\operatorname{int}(H) \neq \varnothing$ and (14) is satisfied. Therefore, from now on we assume that $\operatorname{int}(R) \cap h_{0} s_{0}=\emptyset$. If $R \cap\left(h_{0} s_{0}-\left\{h_{0}, s_{0}\right\}\right) \neq \varnothing$ then we have only two possibilities:

- $h_{0} s_{0}$ and $r_{1} r_{2}$ are parallel. In this case $h_{0} s_{0}$ must be contained in the line $r_{1} r_{2}$ and you can check that either (a) $s_{1} \in \operatorname{int}(R)$, (b) $z_{2} \in \operatorname{int}(H)$ or (c) $s_{1}=z_{2}$ and $t_{1} \in \operatorname{int}(R)$.

- $h_{0} s_{0}$ and $r_{1} r_{2}$ are not parallel. In this case, since we are assuming that $r_{0} r_{1}$ and $h_{0} h_{1}$ are not parallel and that $\operatorname{int}(R) \cap h_{0} s_{0}=\emptyset$, there is a vertex $r_{i} \in h_{0} s_{0}-\left\{h_{0}, s_{0}\right\}$ and (15) implies that $z_{i} \in \operatorname{int}(H)$.

Thus, if $R \cap\left(h_{0} s_{0}-\left\{h_{0}, s_{0}\right\}\right) \neq \varnothing$ then (14) is satisfied and we can assume that

$$
R \cap\left(h_{0} s_{0}-\left\{h_{0}, s_{0}\right\}\right)=\emptyset .
$$


Now let us analyze the intersections of $R$ with the side $h_{3} h_{0}$ of $H$. Since this side is populated with the sentinels $h_{3}, s_{3}, x_{i}$ and $h_{0}$, which are at most $\delta$ apart, Lemma 1 yields that if $\operatorname{int}(R) \cap h_{3} h_{0} \neq \emptyset$ then we have a situation like the one in the right side of Figure 4, with $q_{k}=h_{3}, q_{k+1}=h_{0}$ and $q_{k+2}=h_{1}$ and $p_{j}$ as one vertex $r_{i}$ of $R$. We claim that in this case $z_{i} \in \operatorname{int}(H)$. In fact, since $\left\|z_{i}-p_{i}\right\|=\delta$ is at least as big as the diameter of $\mathcal{D}$ we have that $z_{i} \notin r_{i} y$. Let $d$ be the point defined in items (iv)-(vi) of Lemma 1. We have this three possibilities:

- $d$ satisfies (iv): This case case contradicts (16) and need not to be considered.

- $d$ satisfies (v). In this case we have that

$$
\left\|r_{i}-d\right\|>\|y-d\| \geq \sup \Delta(Q) \geq \delta=\left\|r_{i}-z_{i}\right\|
$$

and $\left\|r_{i}-d\right\|>\delta$. This inequality combined with $z_{i} \notin z_{i} y$ and $\left\|z_{i}-r_{i}\right\|=\delta$ implies that $z_{i} \in y d-\{y, d\} \subset \operatorname{int}(H)$. Thus, $z_{i} \in \operatorname{int}(H)$ in case (v).

- $d$ satisfies (vi): In this case $d \in h_{0} h_{1} \cap R$ and since $R \cap\left(h_{0} s_{0}-\left\{h_{0}, s_{0}\right\}\right)=\emptyset$ we must have $d \in s_{0} h_{1}$. This implies that $\left\|d-h_{0}\right\| \geq \delta$ and, since $q_{k+1}=h_{0}$ in our context, (iv) implies that $\|y-d\| \geq \delta$. We can then use the same argument following (17) and conclude that $z_{i} \in \operatorname{int}(H)$.

In summary, we have shown that if $\operatorname{int}(R) \cap h_{3} h_{0} \neq \emptyset$ then (14) holds. Let us then assume that $\operatorname{int}(R) \cap h_{3} h_{0}=\emptyset$. Using this assumption and (16) it is easy to show that if $R \cap\left(h_{3} h_{0}-\left\{h_{3}, h_{0}\right\}\right) \neq \emptyset$ then there exists $r_{i} \in\left(h_{3} h_{0}-\left\{h_{3}, h_{0}\right\}\right)$ and $z_{i} \in \operatorname{int}(H)$. Thus, we can assume that $R \cap\left(h_{3} h_{0}-\left\{h_{3}, h_{0}\right\}\right) \neq \emptyset$. This assumption, the fact that $\operatorname{int}(H) \cap \operatorname{int}(R) \neq \emptyset$ and (16) imply that $R \cap\left(h_{3} h_{0} \cap\right.$ $\left.h_{0} s_{0}-\left\{h_{3}, s_{0}\right\}\right)=\emptyset$. Using symmetry we can resume our conclusions up to this point as the statement that either

$$
R \cap\left(h_{3} h_{0} \cup h_{0} s_{0} \cup h_{1} h_{2} \cup h_{2} s_{2}-\left\{h_{3}, s_{0}, h_{1}, s_{2}\right\}\right)=\emptyset
$$

or (14) is satisfied and we assume (18) from now on.

Equation (18) shows that if $\left\{r_{0}, r_{1}, r_{2}, r_{3}\right\} \cap \operatorname{int}(H)=\emptyset$ and $\operatorname{int}(H) \cap \operatorname{int}(R) \neq$ $\emptyset$ then one of the sides $r_{0} r_{1}$ or $r_{2} r_{3}$ crosses both segments $s_{0} h_{1}$ and $s_{2} h_{3}$, 
at points $e$ and $f$, say. Since $\|f-e\|>\delta$ we conclude that if $R$ is "short", i.e., if $b(R) \leq 2 \delta$, then $\left\{z_{0}, s_{2}\right\} \cap \operatorname{int}(H) \neq \emptyset$. Therefore, we can assume that

$$
b(R)>2 \delta \quad \text { and } \quad\left\{r_{0}, r_{1}, r_{2}, r_{3}, z_{0}, z_{2}\right\} \cap \operatorname{int}(H)=\emptyset
$$

and that $z_{0}$ is on or above the line $h_{2} h_{3}$ and $z_{2}$ is on or below the line $h_{0} h_{1}$ (These details are illustrated in Fig. 10.) We then have our three final possibilities:

(i) If $r_{0} r_{1} \cap\left(s_{0}^{\prime} h_{3}-\left\{s_{0}^{\prime}\right\}\right) \neq \emptyset$ then $R$ must be inclined to the left as illustrated in Figure 10, because $r_{0} r_{1} \cap h_{0} s_{0}-\left\{s_{0}\right\}=\emptyset$. As a result, the point $\bar{z}_{0}$ is above $h_{2} h_{3}$ and $\bar{z}_{0}$ is below $h_{0} h_{1}$ and if $e f=\bar{z}_{0} \bar{z}_{1} \cap H$ then $\|e-f\|>\delta$. Since the segment $e f-\{e, f\}$ is populated with sentinels $w_{i}$ which are less $\delta$ apart and $\left\|\bar{z}_{0}-w_{1}\right\|<\delta$ and $\left\|\bar{z}_{2}-w_{n_{w}}\right\|<\delta$ at least one $w_{i}$ belongs to $\operatorname{int}(H)$ and (14) is satisfied.

(ii) The case $r_{2} r_{3} \cap\left(s_{2}^{\prime} h_{1}-\left\{s_{2}^{\prime}\right\}\right) \neq \emptyset$ is symmetric to item (i).

(iii) If $r_{0} r_{1} \cap\left(s_{0}^{\prime} h_{3}-\left\{s_{0}^{\prime}\right\}\right)=\emptyset$ and $r_{2} r_{3} \cap\left(s_{2}^{\prime} h_{1}-\left\{s_{2}^{\prime}\right\}\right)=\emptyset$ then we are in the situation described on the right of Figure 10. In this case we can exchange $H$ and $R$ and repeat the argument in item (i).

\section{Concluding remarks}

We defined the concept of sentinels and found finite sentinel assignments for finite families of polygons with internal angles bigger than or equal to $\pi / 2$. We presented optimal assignments for some families of rectangles. It would be interesting to characterize optimal sentinel assignments for more general families of polygons. For instance, the sentinel assignments for the rectangles suggest that by locating sentinels along well chosen lines in the interior of the polygons it is possible to produce smaller sentinel assignments.

The concept of sentinel provides a new approach, based on nonlinear programming, for solving a large variety of packing problems to optimality. Defining sentinels for a given set of polygons may be a hard task. Modelling the problem of finding the sentinels set as a mathematical programming problem will be the subject of future research. It may allow the straightforward application of sentinels-based packing techniques on real applications. 


\section{Acknowledgments}

We would like to thank Professor I. Bárány for his suggestions and for encouraging us to improve our initial work on sentinels and obtain the proofs we present in this work.

\section{REFERENCES}

[1] E.G. Birgin, J.M. Martínez, W.F. Mascarenhas and D.P. Ronconi, Method of sentinels for packing items whitin arbitrary convex regions. Journal of the Operational Research Society, 57 (2006), 735-746.

[2] H. Edelsbrunner, Geometry and Topology for Mesh Generation. Cambridge Univ. Press, England (2001).

[3] G. Fejes Tóth, Densest Packing of Typical Convex Sets are Not Lattice-Like. Discrete \& Computational Geometry, 14 (1995), 1-8.

[4] M. van Kreveld, On fat partition, fat covering and the union size of polygons. Computational Geometry Theory and Applications, 9 (1998), 197-210.

[5] A. Mahadevan, Optimization in computer aided pattern packing. Ph.D. Thesis, North Carolina State University (1984).

[6] V.J. Milenkovic, Rotational polygon containment and minimum enclosure using only robust 2D constructions. Computational Geometry, 13 (1999), 3-19.

[7] Yu G. Stoyan, On the generalization of the dense allocation function. Reports Ukrainian SSR Academy of Sciences, Ser. A., 8 (1980), 70-74 (in Russian).

[8] Yu, G. Stoyan, M.V. Novozhilova and A.V. Kartashov, Mathematical Model and method for searching a local extremum for the non-convex oriented polygons allocation problem. European Journal of Operational Research, 92 (1996), 193-210. 